Proceedings of the 9th National Symposium of Synchrotron Radiation Users, Warsaw, September 26-27, 2011

\title{
Characterisation of the Defect Structure in Gadolinum Orthovanadate Single Crystals Grown by the Czochralski Method
}

\author{
E. WierzbickA ${ }^{a}$, A. Malinowska $^{a *}, \mathrm{~K}$. WieteskA $^{b}$, W. Wierzchowski ${ }^{a}$, \\ M. Lefeld-Sosnowska ${ }^{c}$, M. Świrkowicz ${ }^{a}$, T. EukasiewiCz ${ }^{a}$, C. Paulmann $^{d}$ \\ ${ }^{a}$ Institute of Electronic Materials Technology, Wólczyńska 133, PL-01919 Warsaw, Poland \\ ${ }^{b}$ National Centre for Nuclear Research, A. Soltana 7, PL-05400 Otwock-Świerk, Poland \\ ${ }^{c}$ Institute of Experimental Physics, University of Warsaw, Hoża 69, PL-00681 Warsaw, Poland \\ ${ }^{d}$ HASYLAB at DESY, Notkestrasse 85, D-22607 Hamburg, Germany
}

\begin{abstract}
The $\mathrm{GdVO}_{4}$ single crystals, both undoped and doped with erbium or thulium, were studied by means of X-ray diffraction topographic methods exploring laboratory and synchrotron radiation sources. Variously developed block structure, caused probably by thermal stresses, was revealed. The highest crystallographic perfection was observed in the crystal doped with 4 at.\% of thulium, which was free of the grain boundaries in the end part. Contrary to that, the differences in structural perfection between samples cut out from various regions of the crystal and for different kinds of doping, were less distinct in other crystals. The diffraction topographic methods enabled the statement that the misorientation between various blocks is in the range of several arc minutes.
\end{abstract}

PACS: $61.72 . \mathrm{Ff}$

\section{Introduction}

Gadolinium orthovanadate $\left(\mathrm{GdVO}_{4}\right)$ belongs to the group of rare earth orthovanadates $\left(\mathrm{REVO}_{4}\right)$, which are promising optical materials for applications in lasers and polarisers. These crystals may be host for some ions responsible for the laser phenomena [1-21]. In particular, the gadolinium orthovanadate is a perspective material for diode-pumped solid-state lasers [1-14].

$\mathrm{GdVO}_{4}$ crystallises in the tetragonal structure of zircon type with space group $I 4_{1} /$ amd and lattice parameters $a=b=7.218 \AA$ and $c=6.356 \AA$ [14]. The crystal structure enables efficient introduction of the required ions by doping.

There are many difficulties associated with growing of high quality $\mathrm{REVO}_{4}$ single crystals. These difficulties are connected mainly with low thermal conductivity of these materials. Such growth defects as cracking, low angle boundaries, dislocations, glide bands, inclusions, and substructure were observed [9-11, 13, 15-20, 22-23]. Also in the case of $\mathrm{GdVO}_{4}$, a tendency for spiral growth manifesting itself through formation of characteristic "tail" is observed [9-11, 13, 15]. Frequently, the unwanted defects significantly limit the possibilities of applications of the given material. Their presence indicates a need of studies of the real crystal structure and lattice defects as a function of the growth conditions, leading to determination of the growth conditions favourable for attaining the desired quality.

The aim of the paper is the investigation of the real crystal lattice structure of $\mathrm{GdVO}_{4}$ single crystals, both

* e-mail: agnieszka.malinowska@itme.edu.pl undoped and doped with erbium or thulium. The studies were realised by means of X-ray diffraction topographic methods, which are very sensitive to crystal lattice deformation. Our former investigation of $\mathrm{YVO}_{4}$ crystal, which belongs to the same group of materials, has been published elsewhere [22-23].

\section{Experiment}

Single crystals of $\mathrm{GdVO}_{4}$ were grown by the Czochralski method using Cyberstar Oxypuller 20-04 equipment. The thermal system consisted of a $50 \mathrm{~mm}$ diameter and $50 \mathrm{~mm}$ high iridium crucible, and a passive iridium afterheater of $60 \mathrm{~mm}$ diameter placed on the ceramics around the top part of the crucible. Inductive heating with Huttinger generator was applied. The following conditions of the growth process have been applied: growth rate $1-2 \mathrm{~mm} / \mathrm{h}$; rotation rate $10-20 \mathrm{rpm}$ and cooling after growth - at least $24 \mathrm{~h}$. The growth atmosphere consisted of nitrogen with $0.5 \mathrm{vol} . \%$ admixture of oxygen. To suppress the "tail" formation and to keep temperature gradient over the melt as stableas possible, the cru-

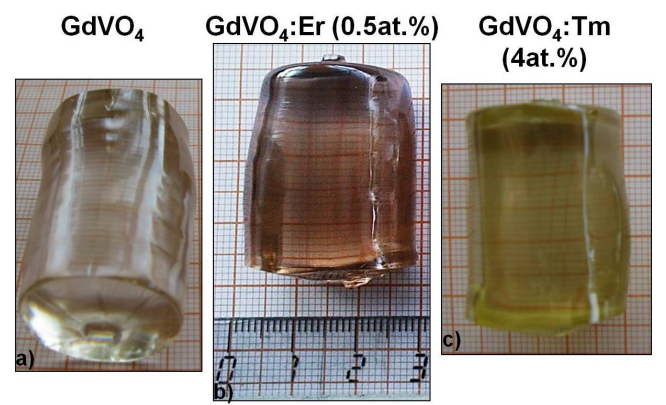

Fig. 1. Examples of as grown $\mathrm{GdVO}_{4}$ crystals. 
cible was lifted up during growth runs to compensate melt level lowering. Finally, good quality, $\langle 100\rangle$-oriented $\mathrm{GdVO}_{4}$ single crystals both undoped and doped with Er (0.5 at.\%) or Tm (4 at.\%) were obtained (Fig. 1). Their diameters and lengths were up to $25 \mathrm{~mm}$ and $50 \mathrm{~mm}$, respectively. The dopant concentrations were determined with the use of inductively coupled plasma-optical emission spectroscopy (ICP-OES) method. According to the obtained data, the distribution coefficients have been estimated as: $k_{\mathrm{Er}} \approx 0.7 ; k_{\mathrm{Tm}} \approx 0.8$ [24]. Therefore, it should be pointed out that erbium and thulium could be relatively easily introduced into $\mathrm{GdVO}_{4}$ lattice.

The samples for X-ray topographic studies were cut out perpendicularly to the [100] growth axis. The samples were taken from different regions of the crystal (near the seed and near the end part), in order to reveal possible changes of the defect structure along the given crystal. All samples were mechanically and chemically polished and thinned down to the thickness of about $300 \mu \mathrm{m}$.

The characterisation of defect structure was performed by X-ray diffraction topographic methods. The conventional Lang projection X-ray topographs were taken in transmission and back-reflection geometry using $\mathrm{MoK} \alpha_{1}$ radiation for different sample azimuths. The topographs were recorded on $50 \mu \mathrm{m}$ Ilford L4 nuclear plates. The Lang projection topographs, due to the translation mechanism, show an image of the whole sample: the thin nearsurface layer in the back-reflection case or whole sample thickness in the transmission case.

The white beam synchrotron radiation (WBSR) backreflection projection and section topographs were taken at low glancing angle of $5^{\circ}$ at the Station F1 at HASYLAB (DESY, Hamburg). The synchrotron projection topographs could reproduce only a limited region of the samples with the diameter close to about $7-8 \mathrm{~mm}$, however, the high collimation of the beam allowed to gain a better image resolution than that obtainable for the Lang topographs. The section topographs were recorded using a narrow beam with the wave front limited by a $5 \mu \mathrm{m}$ slit. The superimposed projection and section topographs were also taken. All synchrotron topographs were recorded on KODAK SR 45 films.

All presented topographs are negative prints, i.e. a higher blackening corresponds to higher X-ray intensity.

\section{Results}

Figures 2-5 display some representative topographs of the investigated $\mathrm{GdVO}_{4}$ crystals. As one can notice, no segregation fringes, and no strong contrasts are observed, indicating a good homogeneity of chemical composition (the contrasts mentioned would indicate the presence of conglomerations of precipitates or dislocations). The Lang topographs reveal several regions with different blackening corresponding to the blocks with different inclination to the sample surface and not exactly set in reflecting position. The effect is shown in Fig. $2 \mathrm{a}-\mathrm{c}$, where back-reflection topographs $2 \mathrm{~b}$ and $2 \mathrm{c}$ were taken for the same reflection but slightly different angular crystal setting, whereas the topograph $2 \mathrm{a}$ was taken in transmis-

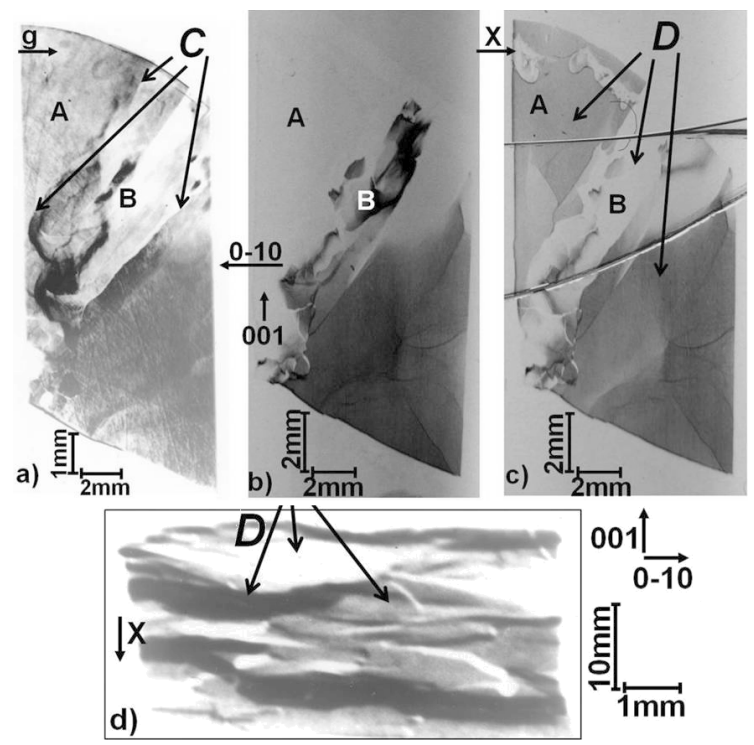

Fig. 2. Lang projection diffraction topographs of the samples cut out from the near seed part (a)-(c) and near end part (d) of the undoped $\mathrm{GdVO}_{4}$ crystal, MoK $\alpha_{1}$ radiation: (a) transmission topograph, 020 reflection; (b)-(c) 10,-4,0 back-reflection topographs taken for different angular crystal setting; the distinct two lines in the middle of the image is caused by the damage of the nuclear plate; (d) back-reflection topograph, 020 reflection. $\boldsymbol{g}$ - diffraction vector; X denotes projection of the incident beam direction on the film; $\mathrm{C}-$ grain boundaries; D - crystal blocks.

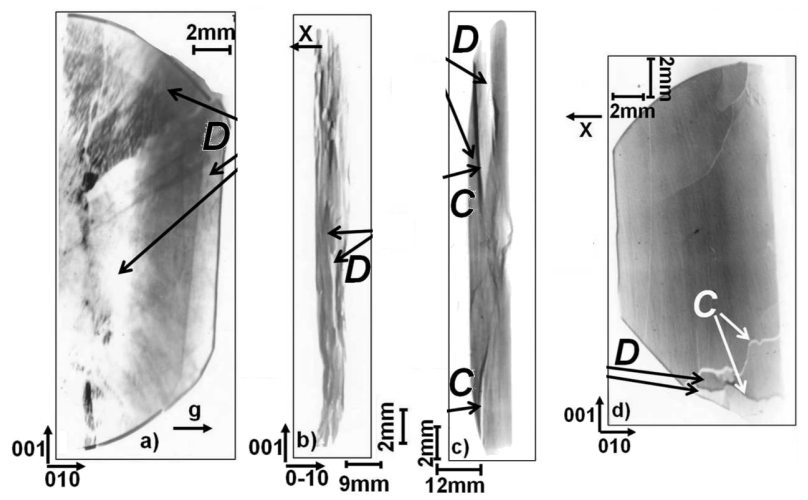

Fig. 3. Lang projection diffraction topographs of the samples cut out from the $\mathrm{GdVO}_{4}: \operatorname{Er}(0.5$ at.\%) (a)-(b) and $\mathrm{GdVO}_{4}: \operatorname{Tm}\left(4\right.$ at.\%) (c)-(d), MoK $\alpha_{1}$ radiation: (a) (c) - samples from the near seed part; (b) (d) - samples from the near end part; (a) transmission topograph (b) - (d) back-reflection topographs. g - diffraction vector; $\mathrm{X}$ denotes projection of the incident beam direction on the film; $\mathrm{C}$ - grain boundaries; D - crystal blocks.

sion geometry. The image of the block marked by A in Fig. 2a and 2c practically disappears in Fig. 2b. Similarly, the image of the blocks marked by $\mathrm{B}$ in Fig. $2 \mathrm{~b}$ practically disappears in Fig. 2a and 2c. The back reflection topograph of the sample cut out from the end part of the crystal shows much more complex block structure (Fig. 2d). 

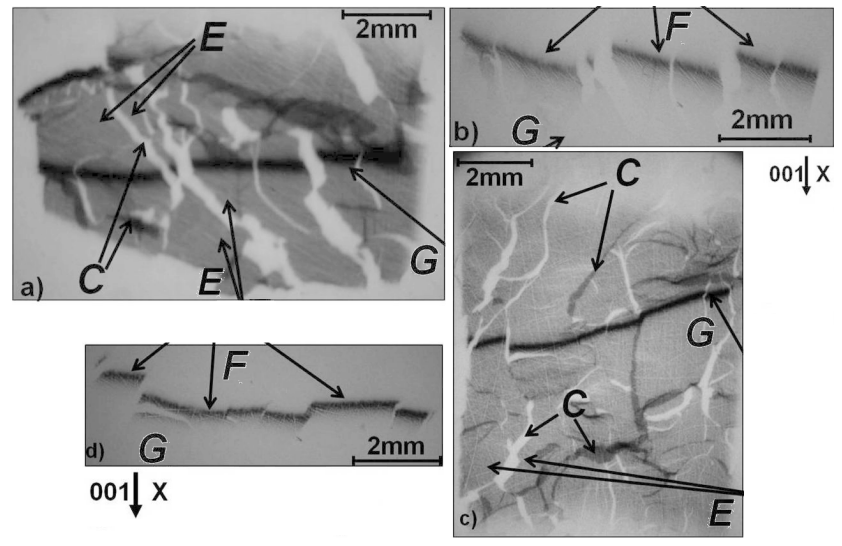

Fig. 4. Synchrotron white beam back-reflection diffraction topographs of the $\mathrm{GdVO}_{4}$ crystals: (a)-(b) - sample cut out from the near end part of the undoped crystal; (c)-(d) sample cut out from the near end part of the crystal doped with $\operatorname{Er}(0.5$ at.\%); (a) (c) - projection topographs with superimposed section topographs (images of the slit); (b), (d) - section topographs. $\mathrm{X}$ denotes projection of the synchrotron radiation beam direction on the film; $\mathrm{C}$ - grain boundaries; $\mathrm{E}$ - displaced images of disoriented blocks; $\mathrm{G}$ image of the linear slit; F - displaced fragments of the slit image corresponding to the various crystal blocks.

In the white beam synchrotron radiation backreflection projection topographs, the disoriented blocks give mutually displaced images with almost the same blackness. They correspond to a diffracted beam of similar intensity but reflected along slightly different directions - each block choosing the appropriate wavelength meeting the Bragg condition (see scheme shown in Fig. 6 and topographs in Figs. 4-5) [25]. The grain boundaries appear as white or black stripes depending on the mutual misorientation between blocks (marked in Figs. 4-5). The black stripes correspond to overlapping of the images from two neighbouring crystal blocks, whereas the white lines correspond to a gap between the images of two blocks (see Fig. 6).

The results indicate that the dominating imperfection in the studied $\mathrm{GdVO}_{4}$ single crystals is a variously developed block structure, which is generally caused by thermal stress. The block structure seems to be much more complex in samples cut out from the end part of the crystal than in those cut out from the near-seed part (Figs. 2, 3a, 3b, 4). The $\mathrm{GdVO}_{4}$ crystal doped with thulium is an exception. In this case, the mosaic structure and other defects are practically absent in the sample cut out from the end part (Figs. 3c-d and 5). The crystal doped with thulium is the most perfect among all $\mathrm{GdVO}_{4}$ crystals studied in this work (Figs. 2-5). This observation was also confirmed by topographs of samples cut out from a crystal grown in another process (Fig. 5eh). The results suggest that the development of the block structure can be moderated by doping, but precise determination of this dependence requires further investi-

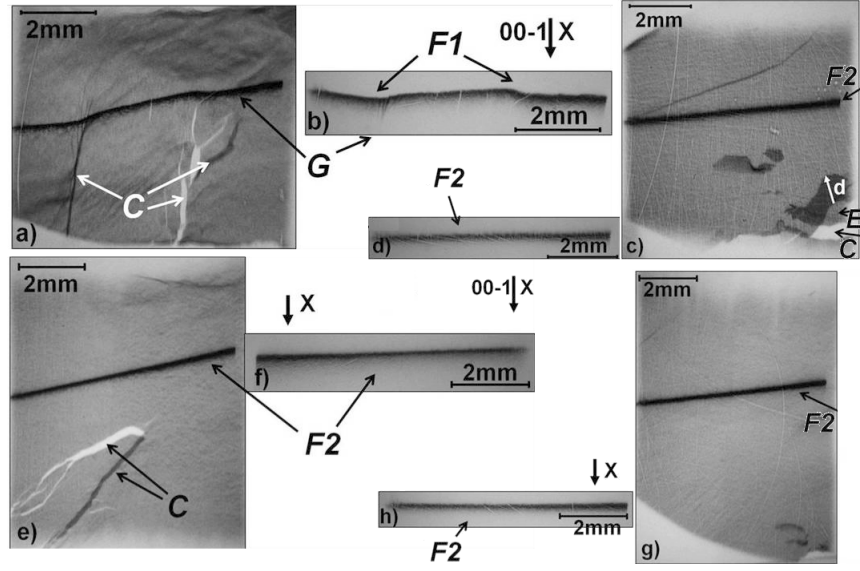

Fig. 5. Synchrotron white beam back-reflection diffraction topographs of two different $\mathrm{GdVO}_{4}$ : Tm (4 at.\%) crystals: (a)-(b) and (e)-(f) - samples cut out from the near seed part of the crystal; (c)-(d) and (g) - (h) - samples cut out from the near end part of the crystal; (a) (c) (e) and (g) - projection topographs with superimposed section topographs (images of the slit); (b) (d) (f) and (h) — section topographs. X denotes projection of the synchrotron radiation beam direction on the film; $\mathrm{C}$ - grain boundaries; $\mathrm{E}$ displaced image of the disoriented block; G - image of the linear slit; F1 - straight segments of the slit image corresponding to slightly disoriented blocks; F2 - practically undeformed image of the slit indicating good quality of the crystal lattice.
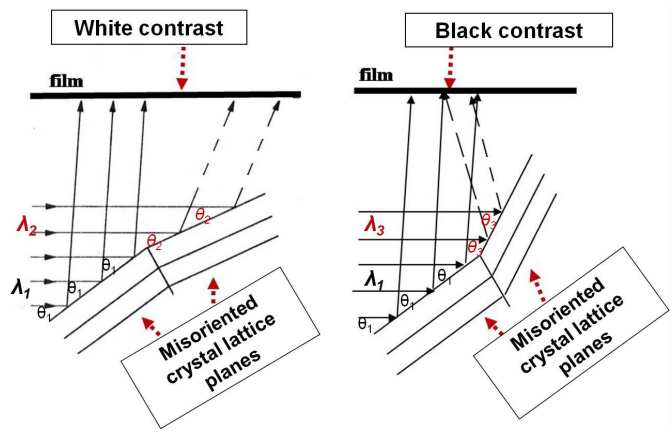

Fig. 6. Model of the orientation contrast formation in the white beam back-reflection synchrotron topographs of the crystals with the block structure. Based on [24].

gations. It is well known that the high concentration of dopant can significantly disturb the glide and multiplication of dislocations, leading to a reduction of the concentration of dislocations in the growing crystals. In particular, a high isoelectronic doping is sometimes used to reduce the concentration of dislocations in $\mathrm{A}^{\mathrm{III}} \mathrm{B}^{\mathrm{V}}$ crystals and GaN layers [26, 27].

The white beam synchrotron radiation back-reflection projection topographs enable a rough evaluation of lattice misorientation between neighbouring blocks. This may be significantly improved by additional exposing of section topographs using a beam limited by a slit. The image of the slit is divided (due to the misorientation of grains present in the illuminated region of the crystal) 
into a few mutually displaced segments, in some cases being also slightly bent (Figs. 4, 5a,b). The evaluated misorientation between various blocks was in the range of several arc minutes.

\section{Conclusions}

The dominating imperfection of the investigated $\mathrm{GdVO}_{4}$ crystals is a variously developed block structure. The block structure is generally caused by thermal stresses, but the development of this structure can also be moderated by some kinds of doping. The evaluated misorientation between various blocks is in the range of several arc minutes.

The results reveal some changes between the samples cut out from different regions of the $\mathrm{GdVO}_{4}$ crystals contrary to our former results in variously doped $\mathrm{YVO}_{4}$ crystals. It was observed that the block structure is much more developed in samples cut out from the end part of the $\mathrm{GdVO}_{4}$ crystals.

The presently observed defects appear to be analogous to those found in Czochralski grown crystals reported in the literature. However, some of them evidently contain dislocations and inclusions at a higher concentration level. On the other hand, in some earlier described orthovanadate crystals obtained by slow cooling method, the concentration of dislocations has been very low.

Some differences between variously doped crystals is indicated. In particular, the best perfection has been observed in the crystal containing thulium at a high concentration level (4 at.\%), where the mosaic structure is practically absent. The results suggest that the development of the block structure can be moderated by doping, but precise determination of this dependence requires further investigations.

\section{Acknowledgements}

The synchrotron investigations were supported by the EC 20100001 HASYLAB project. The technical assistance of J. Bondziul (Warsaw University) is highly appreciated.

\section{References}

[1] E.A. Maunders, L.G. DeShaser, J. Opt. Soc. 61, 684 (1971).

[2] G. Will, W. Schäfer, J. Phys. C (Solid State Phys.) 4, 811 (1971).

[3] M. Bass, IEE J. Quantum Electr. QE-11, 938 (1975).

[4] A.I. Zagumennyi, V.G. Ostroumov, I.A. Shcherbakov, T. Jensen, J.P. Meyen, G. Huber, Sov. J. Quantum Electr. 22, 1071 (1992).

[5] K. Shimamura, S. Uda, V.V. Kochurikhin, T. Taniuchi, T. Fukuda, Jpn. J. Appl. Phys. 35, 1832 (1996).

[6] S. Erdei, B.M. Jin, F.W. Ainger, B. Kezel, J. Vandlik, A. Suveges, J. Cryst. Growth 172, 466 (1997).
[7] C.Q. Wang, H.J. Zhang, Y.T. Chow, J.H. Liu, L. Zhu, J.Y. Wang, X.L. Meng, W.A. Gambling, Opt. Laser Technol. 33, 439 (2001).

[8] H.D. Jiang, H.J. Zhang, J.Y. Wang, H.R. Xia, X.B. Hu, B. Teng, C.Q. Zhang, Opt. Commun. 198, 447 (2001).

[9] L. Qin, X. Meng, J. Zhang, L. Zhu, H. Zhang, B. Xu, H. Jiang, J. Cryst. Growth 242, 183 (2002).

[10] T. Shonai, M. Higuchi, K. Kodaira, T. Ogawa, S. Wada, H. Machida, J. Cryst. Growth 241, 159 (2002).

[11] W. Xiong, S. Lin, Y. Xie, J. Cryst. Growth 263, 353 (2004).

[12] Y. Yang, B. Yao, B. Chen, C. Wang, Opt. Mater. 29, 1159 (2007)

[13] S. Lin, W. Xiong, L. Li, Y. Xie, J. Cryst. Growth 270, 133 (2004).

[14] G. Zhang, H. Tu, Y. Liu, Z. Hu, J. Cryst. Growth 311, 912 (2009).

[15] V.V. Kochurikhin, K. Shimamura, T. Fukuda, J Cryst. Growth 151, 393 (1995).

[16] W. Paszkowicz, P. Romanowski, J. Bak-Misiuk, W. Wierzchowski, K. Wieteska, W. Graeff, R.J. Iwanowski, M.H. Heinonen, O. Ermakova, H. Dąbkowska, Radiat. Phys. Chem. 80, 1001 (2011).

[17] S. Zhao, H. Zhang, J. Liu, J. Wang, X. Xu, Z. Zhao, J. Xu, M. Jiang, J. Cyst. Growth 279, 146 (2005).

[18] B.Q. Hu, Y.Z. Zhang, X. Wu, X.L. Chen, J. Cryst. Growth 226, 511 (2001).

[19] D.E. Eakins, J.B. LeBret, M.G. Norton, D.F. Bahr, J. Cryst. Growth 266, 411 (2004).

[20] J.B. LeBret, M. Grant Norton, D.F. Bahr, D.P. Field K.G. Lynn, J. Mater. Sci. 40, 3347 (2005).

[21] M. Higuchia, T. Shimizua, J. Takahashia, T. Ogawab, Y. Uratac, T. Miurab, S. Wadab, H. Machida, J. Cryst. Growth 283, 100 (2005).

[22] K. Wieteska, W. Wierzchowski, E. Wierzbicka, A. Malinowska, M. Lefeld-Sosnowska, T. Łukasiewicz, W. Graeff, Acta Phys. Pol. A 114, 455 (2008).

[23] A. Malinowska, E. Wierzbicka, M. Lefeld-Sosnowska, K. Wieteska, W. Wierzchowski, T. Eukasiewicz, M. Swirkowicz, W. Graeff, Acta Phys. Pol. A 117, 328 (2010)

[24] A. Karaś, private information (2008).

[25] D.K. Bowen, B.K. Tanner, High resolution $X$ ray diffractometry and topography, London: Taylor\&Francis Ltd., 221 (1998).

[26] X.A. Zhu, C.T. Tsai, J. Appl. Phys. 88, 2295 (2000).

[27] M.A. Moram, M.J. Kappers, F. Massabuau, R.A. Oliver, C.J. Humphreys, J. Appl. Phys. 109 , 073509 (2011). 